\title{
Imaging with Waves in Random Media
}

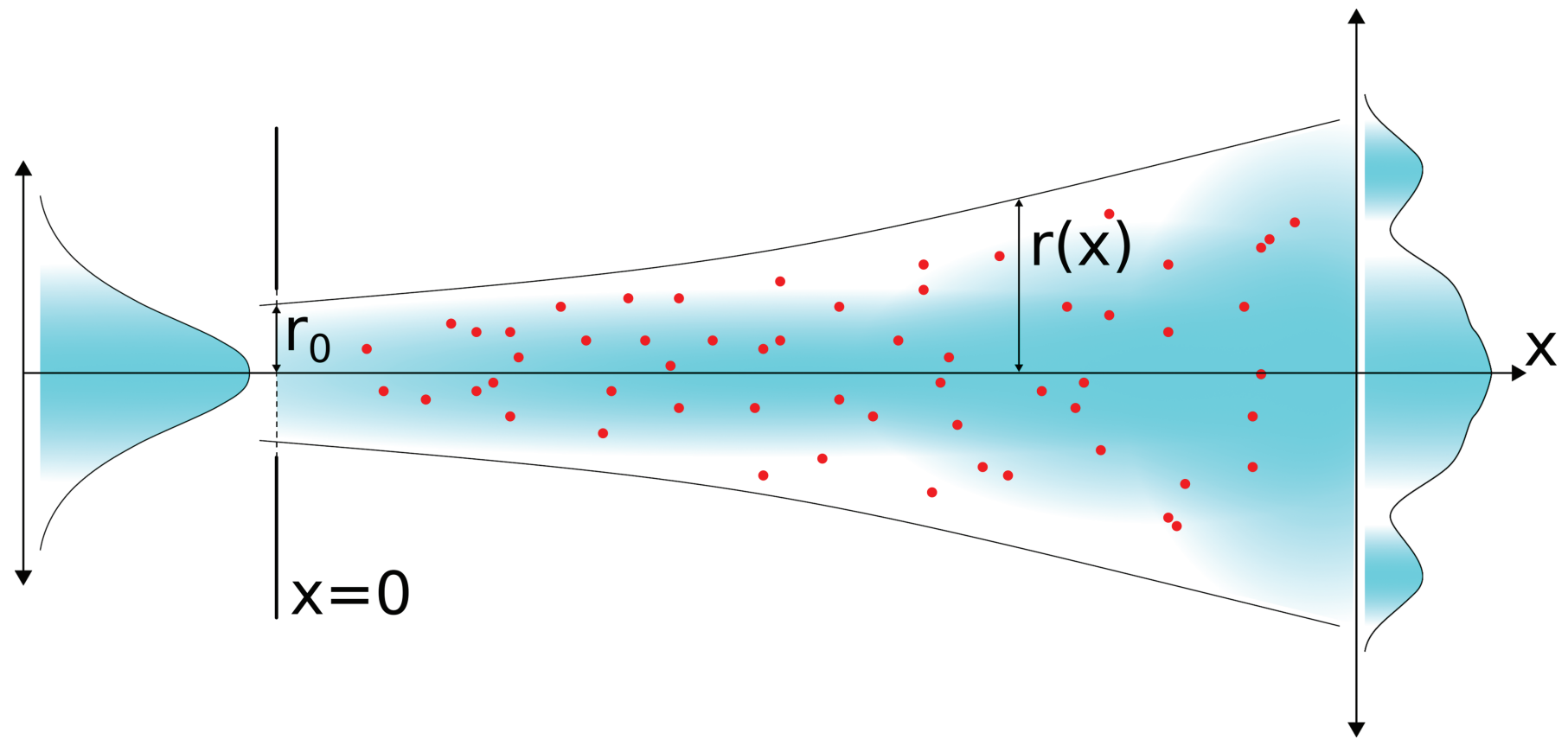

\section{Liliana Borcea}

\section{Introduction}

Wave-based imaging is an inverse problem for the wave equation, with a broad spectrum of applications in nondestructive testing, ultrasound for medical diagnosis, radar, underwater sonar, space surveillance, geophysical exploration, etc. Any imaging system uses sensors that measure sound, electromagnetic, or elastic waves generated by impulsive sources or by ambient noise sources. These waves carry information about the medium through which they travel and their initial state, and the goal of imaging is to extract this information from the sensor measurements, a.k.a. the data.

The sensor configurations are application specific. The illustrations in Figure 1 show sensors that are closely spaced in a bounded set, so for imaging purposes they can be treated as a collective entity called an array. The

Liliana Borcea is the Peter Field Collegiate Professor of Mathematics at the University of Michigan Ann Arbor. Her email address is borcea@umich. edu. Communicated by Notices Associate Editor Reza Malek-Madani.

For permission to reprint this article, please contact: reprint-permission@ams.org.
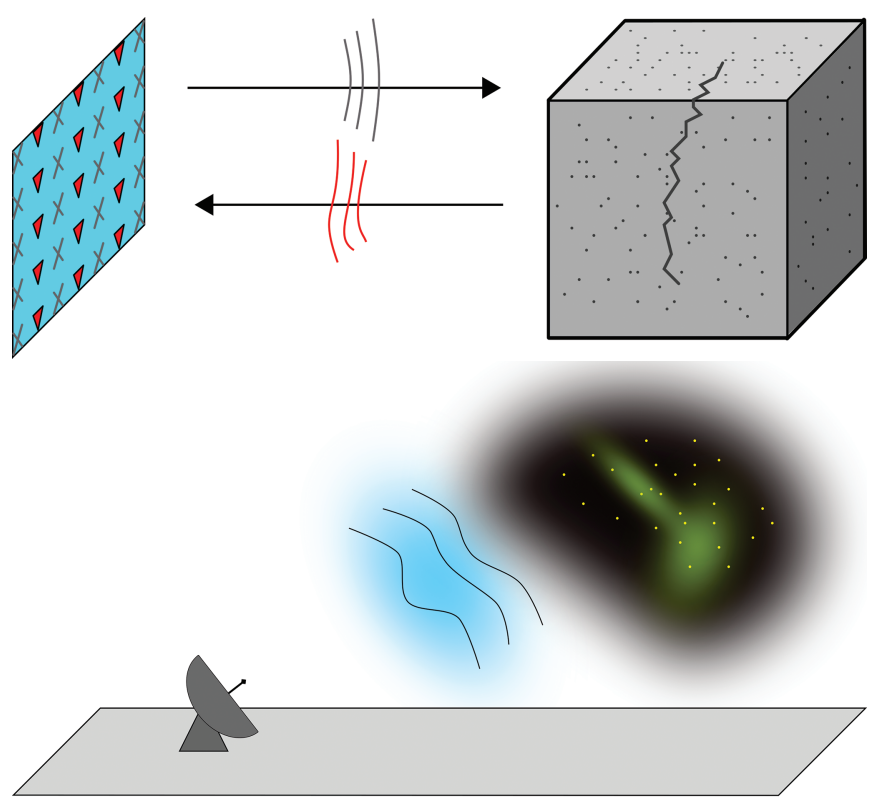

Figure 1. Top: In nondestructive testing an active array of sensors probes a heterogeneous body with waves and records the scattered returns. Bottom: A telescope (passive array of sensors) records waves from astronomical sources. 


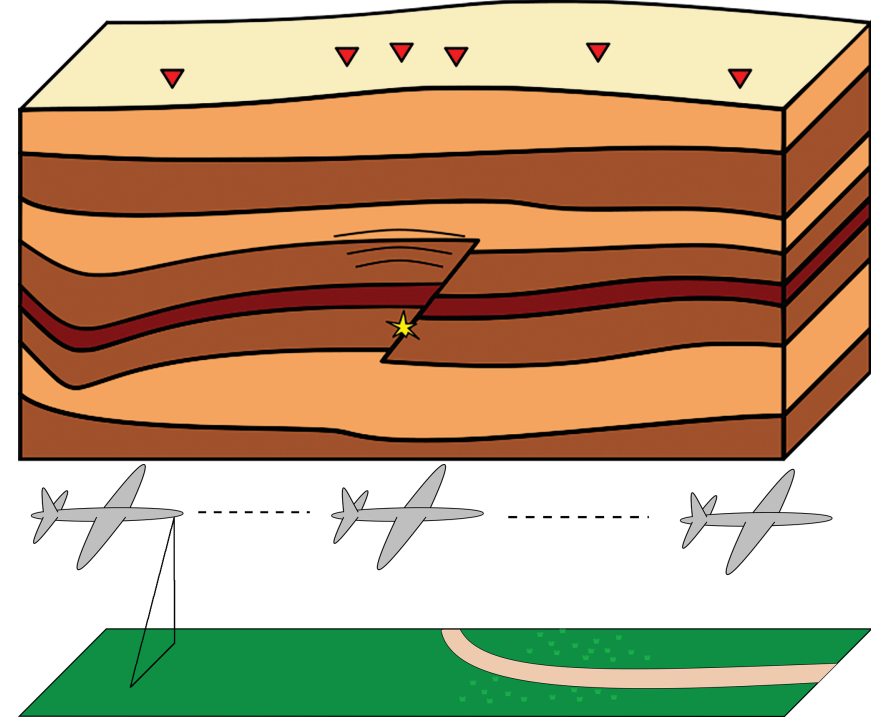

Figure 2. Top: In seismic imaging geophones (red triangles) at the surface of the earth record waves due to an earthquake (yellow star). Bottom: In synthetic aperture radar an antenna mounted on an airplane emits waves and records the returns from the ground.

sensors may act as both impulsive sources and receivers, in which case the array is called active. If the sensors are only receivers, the array is called passive. The configuration shown in the top plot of Figure 2 is typical of seismic imaging, where the sensors are widely spaced geophones lying on the surface of the earth and the sources are uncontrolled, e.g., earthquakes or ambient seismic noise. The configuration shown in the bottom plot of Figure 2 is used in synthetic aperture radar, where the sensors are antennas that emit and receive electromagnetic waves. There may be a single sensor or a small array of sensors mounted on a moving platform that defines a virtual (synthetic) aperture.

Conventional imaging modalities use impulsive sources and operate under the hypothesis that the medium has a smooth and slowly varying wave speed $c$, with the possible exception of a few unknown localized anomalies, such as large jump discontinuities corresponding to reflective structures, modeled by a reflectivity function $\rho$. This hypothesis is used in the forward model, which defines the mapping from $\rho$ and the source $S$, if it is unknown, to the wave at the sensors. The forward mapping is inverted in some approximate sense by data processing, and the result is an imaging function, an image in short, which is displayed over a search domain. The peaks of the image are interpreted as points in the support of the unknown $\rho$ and $S$. The expected sharpness of the peaks is quantified by a resolution theory, which takes into account the bandwidth $B$ and modulation frequency $\omega_{c}$ of the source signal, the typical distance $L$ traveled by the waves, the placement of the sensors, and the aperture ${ }^{1} a$. With the above hypothesis standing, well-known resolution formulas $[8$, Chapter 8] predict image improvement with the increase of $\omega_{c}, B$, and the ratio $a / L$. This has motivated, for example, a new generation of high-frequency and broadband radar systems like the Space Fence program, which operates in the S-band frequency range ( 2 to $4 \mathrm{GHz}$ ) and even Ka-band ( $35 \mathrm{GHz}$ ) and W-band $(95 \mathrm{GHz})$ radars.

Imaging gets quite complicated when the hypothesis of slowly varying wave speed is inadequate. All media have microstructures, which in most imaging applications can be modeled by small amplitude fluctuations of the coefficients $^{2}$ of the wave equation over short length scales compared to $L$. Think of the turbulent atmosphere, complex biological tissue, the earth's crust, or heterogeneous materials like aging concrete. Low-frequency waves are insensitive to such small and rapid fluctuations. They propagate as in an effective medium with constant or slowly and smoothly changing wave speed. However, high-frequency waves interact with the microstructure, and the net effect, called cumulative scattering, builds up over a long enough distance $L$ to cause strong distortion of the wave (see Figure 3). Conventional imaging methods do not account for this distortion and, as a result, they produce unfocused images with spurious peaks that are difficult to interpret. Imaging with waves in random media is concerned with developing methods that mitigate the wave distortion and produce informative images. It is an interdisciplinary field rooted in the physics of wave scattering, where ideas from analysis, probability, scientific computing, experimental science, signal processing, and statistics result in novel imaging modalities.

Why random media? We image in one medium, which typically does not change over the duration of the experiment, so there is not a statistical ensemble of realizations. The point is that the fluctuating coefficients of the wave equation are not known, and it is impossible to estimate them pointwise as part of imaging. In most cases it is not even interesting to determine their small fluctuations. For example, in nondestructive evaluation of a concrete wall, the goal is to find strong reflecting structures, like cracks, and not the pebbles (microstructure) in the concrete.

The random medium is a model of the uncertainty of the fluctuations of the coefficients of the wave equation, whose solution, the wave, is a random field. It is impossible to calculate its probability distribution because the mapping from the random coefficients to the solution is complicated and nonlinear. The theory of wave propagation in random media uses stochastic asymptotic

\footnotetext{
${ }^{1}$ In array imaging, the aperture is the diameter of the bounded set that supports the sensors. In synthetic aperture imaging, it is the length of the path of the moving sensor.

${ }^{2}$ There may also be rough boundaries, which we do not address in this article.
} 

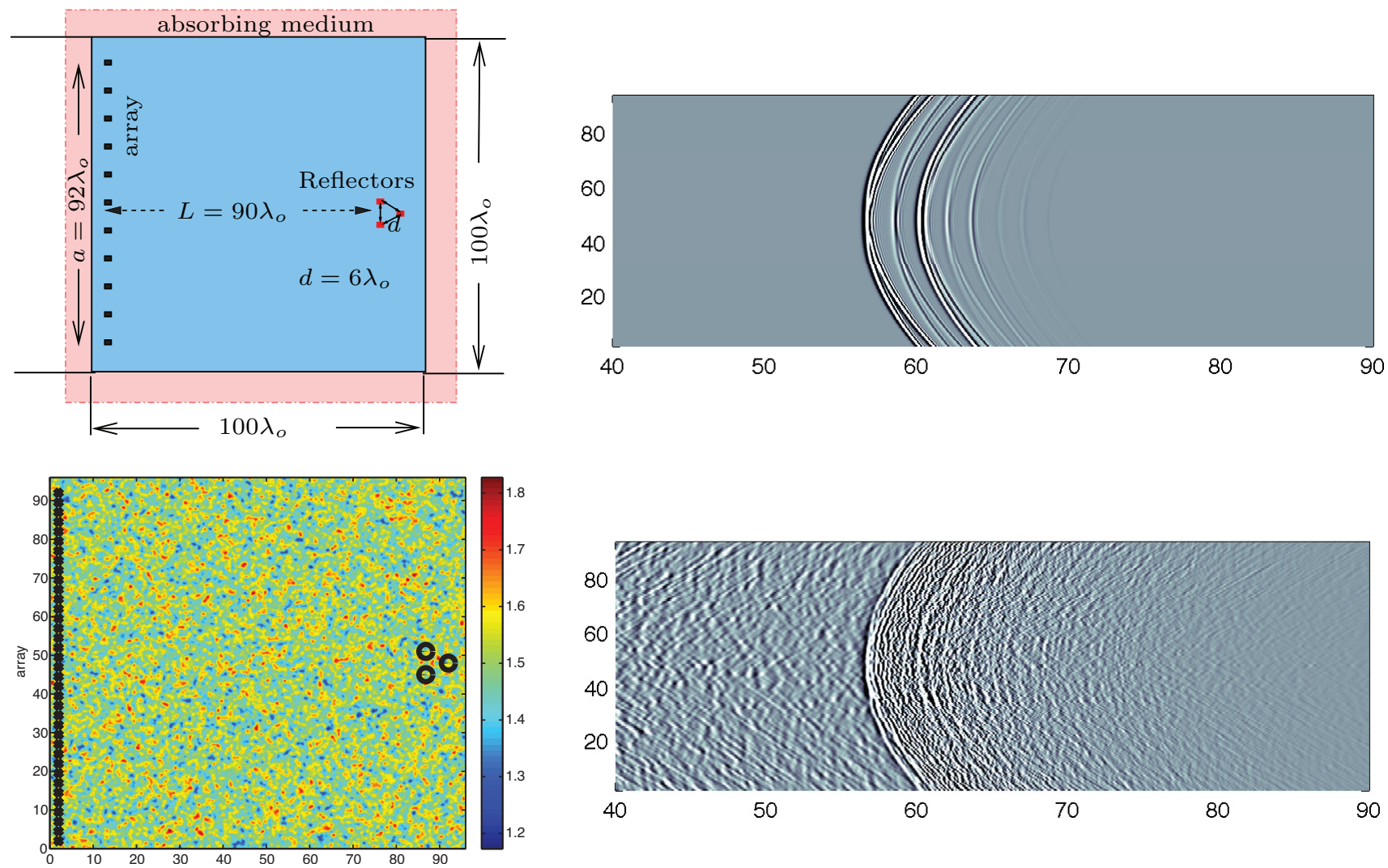

Figure 3. Left plots: Active array probes a medium with three reflectors by emitting a pulse from the central sensor. Right plots: Amplitude of the wave received at the array vs. time on the abscissa, in units of the pulse width, and receiver location on the ordinate, in units of central wavelength $\lambda_{c}$. The echoes from the reflectors are clearly seen when the medium is homogeneous (top right). The cumulative scattering effect of the fluctuations of $c$ (bottom left) causes wave distortion (bottom right). See Acknowledgments section.

analysis to find approximate descriptions of the statistics of the wave in various application-relevant regimes that are defined by the strength of the random fluctuations and the relations between the important length scales: the wavelength, the correlation length of the fluctuations, and the travel distance.

The study of waves in random media has a long history, starting from the 1960s $[16,20]$. A good overview of the field and its early applications is in [15]. Lately, new concepts driven by imaging in random media have emerged. In this article we discuss two of them:

1. Statistical stability, which is a central concept in the resolution theory of imaging in random media [4,7]. It states that although the image is computed from data gathered in one realization of the medium, without ensemble averaging, it should depend only on the statistics of the medium and not the realization. That is to say, the image should be self-averaging.

2. Statistically stable images can be calculated from crosscorrelations of the waves measured at the sensors, even when the sources are uncontrolled, like ambient noise sources.
To introduce these concepts, we consider scalar (sound) waves modeled by the time $t$ and location $\vec{x}$ dependent acoustic pressure $P$, the solution of the wave equation

$$
\begin{array}{r}
\frac{1}{c^{2}(\overrightarrow{\boldsymbol{x}})} \partial_{t}^{2} P(\overrightarrow{\boldsymbol{x}}, t)-\Delta_{\overrightarrow{\boldsymbol{x}}} P(\overrightarrow{\boldsymbol{x}}, t)=S(\overrightarrow{\boldsymbol{x}}, t), \\
t \in \mathbb{R}, \overrightarrow{\boldsymbol{x}} \in \mathbb{R}^{3},
\end{array}
$$

with initial condition $P(\vec{x}, t) \equiv 0$ prior to the excitation from the source $S$. We focus mostly on the problem of imaging the source in the medium with wave speed

$$
c(\overrightarrow{\boldsymbol{x}})=c_{o}[1+\mu(\overrightarrow{\boldsymbol{x}})]^{-\frac{1}{2}},
$$

where the background speed $c_{o}$ is a known constant and $\mu$ is a mean zero stationary random process with ergodic properties. We also comment on the problem of imaging a reflectivity $\rho$, where the variable coefficient in (1) is replaced by $\rho+c^{-2}$, with $c$ given in (2). Note that the mapping $S \mapsto P$ is linear, whereas the mapping $\rho \mapsto P$ is nonlinear. Thus, it is easier to image sources than reflectors. Nevertheless, both problems are complicated by the nonlinear mapping $\mu \rightarrow P$ that models the distortion of the wave. 
In some applications, like geophysical exploration, the background speed $c_{o}$ is not constant but an unknown smooth and slowly changing function that must be estimated via so-called velocity analysis. This involves either nonlinear optimization using large and often redundant data sets or tomographic methods based on measurements of travel times of the wave transmitted through the medium. Velocity analysis is outside the scope of this article, but we discuss briefly how correlations and noisy ambient sources can be used in this context.

The analysis of various imaging modalities requires the calculation of statistical moments of $P$. The first moment is the expectation $\mathbb{E}[P]$, which models the coherent (unscattered) wave. Conventional imaging assumes that $P$ has negligible fluctuations about the mean $\mathbb{E}[P]$ and furthermore that $\mathbb{E}[P]$ is approximately the solution $P_{o}$ of the wave equation with wave speed $c_{o}$. However, it is well known that $\mathbb{E}[P]$ decays at an exponential rate with the travel distance, while the random fluctuations $P-\mathbb{E}[P]$ gain strength [15].

Imaging with cross-correlations becomes interesting when the waves travel deep enough in the medium so that $\mathbb{E}[P] \approx 0$. The expectation of the cross-correlations is determined by the second-order moments of $P$. These are related to the mean Wigner transform, which satisfies transport equations that have been derived in a variety of regimes in $[1,2,13,19]$. The analysis of the random fluctuations of the cross-correlations involves fourth-order moments of $P$. It is not known how to compute these moments, except for a few wave-scattering regimes. One such regime is described by geometrical optics, where the waves travel along deterministic rays but the travel time has random fluctuations [20, Chapter 6]. This weak scattering regime is assumed in the adaptive optics imaging modality and has been used in [4] to study imaging with crosscorrelations. We consider another, stronger scattering regime, called white-noise paraxial, which is relevant for laser beam propagation in random media. Back-scattering is negligible in this regime, and the wave is described by the solution of an Itô-Schrödinger equation [13]. This is an evolution problem along the direction of propagation, the axis of the laser, where Ito stochastic calculus allows the closure of the hierarchy of equations for the moments [11]. Still, without additional assumptions, the fourth-order moments can only be approximated numerically [15, Section 20$]$.

In the next section we describe imaging in the white noise paraxial regime under some additional scaling assumptions that allow explicit fourth-order moment formulas [14]. We use it to present the coherent interferometric (CINT) approach for imaging in random media [7]. In the section "Imaging with Noisy Sources" we discuss the use of cross-correlations for imaging with noisy sources. We end with some "Final Remarks."

\section{Coherent Interferometric Imaging}

In this section we present imaging in random media in a scattering regime where the wave generated by a pulsed source $S$ propagates as a beam along the "range" direction $x$ (Figure 4 ). To write explicit formulas, we use a Gaussian beam model [8, Chapter 4$]$ described in the system of coordinates $\overrightarrow{\boldsymbol{x}}=\left(x, \boldsymbol{x}_{\perp}\right)$ with origin at the center of the source, where the "cross-range" vector $x_{\perp} \in \mathbb{R}^{2}$ lies in the plane orthogonal to the range (beam) axis $x$. The source is localized at $x=0$, with Gaussian profile in the cross-range plane

$$
\begin{aligned}
S(\overrightarrow{\boldsymbol{x}}, t) & =f(t) e^{-i \omega_{c} t} \exp \left(-\frac{\left|\boldsymbol{x}_{\perp}\right|^{2}}{r_{0}^{2}}\right) \delta(x), \\
f(t) & =\exp \left(-\frac{B^{2} t^{2}}{2}\right),
\end{aligned}
$$

where $r_{0}$ is the initial radius of the beam and $\delta$ denotes the Dirac delta distribution. The emitted pulse has Gaussian envelope $f$ and modulation frequency $\omega_{c}$. Its Fourier transform

$$
\begin{array}{r}
\int_{\mathbb{R}} d t e^{i \omega t} f(t) e^{-i \omega_{c} t}=\hat{f}\left(\omega-\omega_{c}\right) \\
=\frac{\sqrt{2 \pi}}{B} \exp \left[-\frac{\left(\omega-\omega_{c}\right)^{2}}{2 B^{2}}\right]
\end{array}
$$

is large in the interval centered at $\omega_{C}$ and of length of order $B$, which is why $\omega_{c}$ is called the central frequency and $B$ is the bandwidth. The duration of the pulse is inversely proportional to $B$, and usually $B \ll \omega_{c}$.

For reference, we begin with a description of the beam in the homogeneous medium, and then summarize the white noise paraxial model of beam propagation in the random medium. This gives the forward map from $S$ to the data gathered at a remote array of receivers located at distance (range) $L$ from the source. This forward map is then used for imaging the source. We also give a brief discussion of imaging reflectors in the random medium.

The reader will note that this section contains many scaling assumptions defined by an asymptotic parameter that describes the order relations between the wavelength, the correlation length of the fluctuations, and the distance of propagation, as well as the amplitude of the fluctuations. These assumptions are rooted in the physics of the problem and are key ingredients of any analysis of wave propagation and imaging, because there is no universal behavior of waves in random media.

Gaussian beam in the homogeneous medium. Let $P_{o}$ denote the wave due to the source (3) in the reference homogeneous medium. This wave is not observed at the sensors, but we give its mathematical expression for the purpose of comparison with the model of the measured 


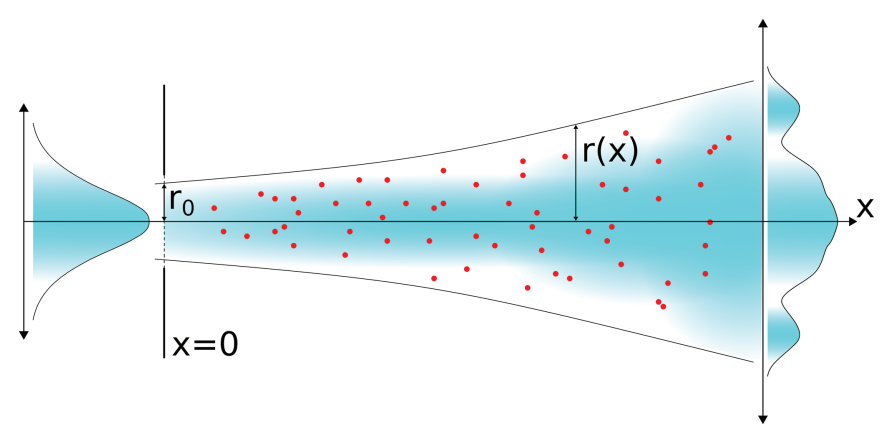

Figure 4. Gaussian beam with initial radius $r_{0}$ that increases to $r(x)$ at range $x$ due to cumulative scattering. The wavefront is also distorted.

wave. This expression is obtained using the assumptions stated in "Scaling relations," which ensure that $P_{o}$ propagates as a beam.

Scaling relations. There are three length scales that influence the evolution of the reference wave $P_{o}$ : the central wavelength $\lambda_{c}=2 \pi c_{o} / \omega_{c}$, the initial radius $r_{0}$, and the range offset $L$ between the receiver sensors and the source. These scales are determined by the design of the imaging system, and in a typical regime of high-frequency beam propagation they satisfy

$$
\lambda_{c} \ll r_{0} \ll L .
$$

It is natural to use the scale separation relation (5) to introduce the dimensionless parameter

$$
\varepsilon=\lambda_{c} / L \ll 1,
$$

so that we can analyze the wave in the asymptotic limit $\varepsilon \rightarrow 0$. It remains to discuss the apparently many choices of the initial radius $r_{0}$ satisfying (5). To understand their effect on the wave $P_{o}$, we turn to the theory of diffraction, which states that

$$
L_{\mathrm{R}} / L \sim r_{0}^{2} /\left(L \lambda_{c}\right),
$$

where the symbol $\sim$ denotes throughout equal, up to an order 1 constant factor, and $L_{\mathrm{R}}$ is the Rayleigh length, the range at which the beam cross-section doubles in area $[8$, Chapter 8]. We conclude from (7) that in order for the wave to propagate as a beam along the range axis $x$, in the limit $\varepsilon \rightarrow 0$, the initial radius must satisfy

$$
r_{0} / L \sim \sqrt{\varepsilon}
$$

so that the ratio (7) is independent of $\varepsilon$. If $r_{0}$ is smaller, then the source is point-like in the limit $\varepsilon \rightarrow 0$ and emits a spherical wave with no privileged direction of propagation. If $r_{0}$ is larger, then the sensors are in the near field of the source as $\varepsilon \rightarrow 0$.

The beam. The solution of (1), in the absence of the fluctuations $\mu$, is approximated by

$$
\begin{aligned}
P_{o}(\overrightarrow{\boldsymbol{x}}, t) & =\int_{\mathbb{R}} \frac{d \omega}{2 \pi} e^{-i \omega t} \hat{P}_{o}(\overrightarrow{\boldsymbol{x}}, \omega), \\
\hat{P}_{o}(\overrightarrow{\boldsymbol{x}}, \omega) & \approx \frac{i \exp [i k(\omega) x)]}{2 k(\omega)} \widehat{u}_{o}\left(x, \boldsymbol{x}_{\perp}, \omega\right),
\end{aligned}
$$

with $k(\omega)=\omega / c_{o}$ the wavenumber at frequency $\omega$. Here $\hat{P}_{o}$ is a monochromatic wave propagating along the $X$ axis, with envelope described in the limit $\varepsilon \rightarrow 0$ by the solution of the Schrödinger equation

$$
\begin{array}{r}
2 i k(\omega) \partial_{x} \hat{u}_{o}\left(x, x_{\perp}, \omega\right)+\Delta_{x_{\perp}} \hat{u}_{o}\left(x, x_{\perp}, \omega\right)=0, \\
x>0, x_{\perp} \in \mathbb{R}^{2},
\end{array}
$$

with initial condition

$$
u_{o}\left(0, x_{\perp}, \omega\right)=\hat{f}\left(\omega-\omega_{c}\right) \exp \left(-\frac{\left|\boldsymbol{x}_{\perp}\right|^{2}}{r_{0}^{2}}\right),
$$

where $\Delta_{\boldsymbol{x}_{\perp}}$ is the Laplacian in the cross-range plane.

The explicit expression of $\hat{u}_{o}$ is known [8, Chapter 5], and substituting it in (9) we obtain

$$
\begin{aligned}
& P_{o}(\vec{x}, t) \approx \frac{i r_{0}}{4 \pi r(x)} \int_{\mathbb{R}} d \omega \frac{\hat{f}\left(\omega-\omega_{c}\right)}{k(\omega)} \\
& \quad \times \exp \left\{-i \omega\left[t-\tau\left(x, x_{\perp}\right)\right]-\frac{\left|\boldsymbol{x}_{\perp}\right|^{2}}{r^{2}(x)}+i \phi(x, \omega)\right\} .
\end{aligned}
$$

This is the Gaussian beam with radius

$r(x)=r_{0}\left[1+\left(\frac{x}{L_{\mathrm{R}}(\omega)}\right)^{2}\right]^{\frac{1}{2}}, \quad L_{\mathrm{R}}(\omega)=\frac{k(\omega) r_{0}^{2}}{2}$,

which grows with $x$ due to diffraction on the range scale quantified by the Rayleigh length $L_{R}$. The beam has the travel time delay

$$
\tau\left(x, x_{\perp}\right)=\frac{1}{c_{o}}\left[x+\frac{\left|x_{\perp}\right|^{2}}{2 X}\right]
$$

and so-called phase retardation

$$
\phi(x, \omega)=\arctan \left[-x / L_{\mathrm{R}}(\omega)\right] \in[-\pi, 0] .
$$

The denominator $X$ in (14) is the range distance from the source to the spherical wavefront passing through $\vec{x}=$ $\left(x, x_{\perp}\right)$. At large $x$ the wavefront is almost planar and $X \approx X$.

Gaussian beam in the random medium. We now consider the random medium with fluctuations of the wave speed (2) modeled by the stationary random process $\mu$ with strong mixing. This satisfies

$$
\mathbb{E}[\mu(\overrightarrow{\boldsymbol{x}})]=0, \quad C\left(\frac{\overrightarrow{\boldsymbol{x}}}{\boldsymbol{\ell}}\right)=\mathbb{E}[\mu(\overrightarrow{\boldsymbol{x}}) \mu(\overrightarrow{\mathbf{0}})],
$$

where $\mathbb{E}$ denotes the expectation. The covariance $C$ of the process $\mu$ is assumed to be an integrable function, at least 
twice continuously differentiable, normalized by

$$
\int_{\mathbb{R}^{3}} d \overrightarrow{\boldsymbol{x}} C\left(\frac{\overrightarrow{\boldsymbol{x}}}{\boldsymbol{\ell}}\right)=\sigma^{2} \ell^{3}, \quad C(\overrightarrow{\mathbf{0}}) \sim \sigma^{2} .
$$

The positive, dimensionless parameter $\sigma \ll 1$ quantifies the small amplitude of the fluctuations, and $\ell$ is the correlation length.

As in the previous section, we begin by stating the scaling assumptions under which the wave generated by the source (3) propagates through the random medium as a beam. Then we give the white noise paraxial model of beam propagation and describe the statistical moments of the wave.

Scaling relations. The random fluctuations $\mu$ introduce the additional length scale $\ell$ and the dimensionless amplitude factor $\sigma$. These are fixed by the medium in which the imaging takes place and cannot be adjusted like $L, r_{0}$, and $\lambda_{c}$ in the design of the imaging system. The scaling relations given here describe the design for which the wave propagates as a beam in the random medium.

We could give the scaling in terms of the dimensionless $\sigma \ll 1$, but in order to connect it to the relations in the previous section we introduce the asymptotic parameter $\varepsilon \ll 1$ by

$$
\sigma=\varepsilon^{3 / 4} \sigma_{0}
$$

where $\sigma_{0}$ is an order 1 constant, i.e., independent of $\varepsilon$. The cumulative scattering of the beam in the random medium is strong when $\ell$ and $r_{0}$ are of the same order in $\varepsilon$. Recalling (8), we choose the range scale

$$
L \sim \frac{\ell}{\sqrt{\varepsilon}} \sim \frac{\ell}{\sigma^{2 / 3}} .
$$

The central wavelength $\lambda_{c}$ is related to $L$ as in (6), and we let the initial radius of the beam satisfy

$$
\frac{\pi L}{L_{\mathrm{R}}\left(\omega_{c}\right)}=\frac{\lambda_{c} L}{r_{0}^{2}} \ll 1 \ll \frac{\lambda_{c} L}{\ell^{2}},
$$

where all the ratios are independent of $\varepsilon$. This means that we can neglect diffraction at the range $L$ of the array in order to showcase the broadening of the beam due to cumulative scattering in the random medium.

We will see that the coherent wave decays on the range scale $S \sim \lambda_{c}^{2} /\left(\ell \sigma^{2}\right)$, called the scattering mean free path [15]. The choice (18) of $\varepsilon$ ensures that the random medium affects the wave at the range $x=L$ of the sensors, because the ratio $L / S$ is fixed as $\varepsilon \rightarrow 0$.

The white noise paraxial model of the beam. It is proved in [13] that under the assumptions (6)-(8) and (18)-(20), the solution of (1) is

$$
\begin{aligned}
P(\vec{x}, t) & =\int_{\mathbb{R}} \frac{d \omega}{2 \pi} e^{-i \omega t} \hat{P}(\vec{x}, \omega), \\
\hat{P}(\vec{x}, \omega) & =\frac{i \exp [i k(\omega) x)]}{2 k(\omega)} \hat{u}^{\varepsilon}\left(x, \boldsymbol{x}_{\perp}, \omega\right),
\end{aligned}
$$

where $\hat{P}$ is a monochromatic wave propagating along $x$, with envelope $\hat{u}^{\varepsilon}$. In the limit $\varepsilon \rightarrow 0, \widehat{u}^{\varepsilon}$ converges in distribution, in $C\left([0, \infty], L^{2}\left(\mathbb{R}^{2}\right)\right)$, to the solution $\hat{u}$ of the Itô-Schrödinger equation

$$
\begin{aligned}
d_{x} \hat{u}\left(x, x_{\perp}, \omega\right)= & {\left[\frac{i}{2 k(\omega)} \Delta_{x_{\perp}} \hat{u}\left(x, x_{\perp}, \omega\right)\right.} \\
& \left.-\frac{k^{2}(\omega)}{8} \mathcal{R}(0) \hat{u}\left(x, x_{\perp}, \omega\right)\right] d x \\
& +\frac{i k(\omega)}{2} \hat{u}\left(x, x_{\perp}, \omega\right) d \mathcal{B}\left(x, x_{\perp}\right),
\end{aligned}
$$

with initial condition (11),

$$
\hat{u}\left(0, x_{\perp}, \omega\right)=\hat{u}_{o}\left(0, x_{\perp}, \omega\right) .
$$

Here $\mathcal{B}$ denotes a Brownian motion field, a Gaussian process independent of $\omega$, satisfying

$$
\begin{aligned}
& \mathbb{E}\left[\mathcal{B}\left(x, x_{\perp}\right)\right]=0, \\
& \mathbb{E}\left[\mathcal{B}\left(x, x_{\perp}\right) \mathcal{B}\left(x^{\prime}, x_{\perp}^{\prime}\right)\right]=\min \left\{x, x^{\prime}\right\} \mathcal{R}\left(x_{\perp}-x_{\perp}^{\prime}\right),
\end{aligned}
$$

with $\mathcal{R}$ defined by the covariance of $\mu$,

$$
\mathcal{R}\left(\boldsymbol{x}_{\perp}\right)=\int_{-\infty}^{\infty} d x C\left(\frac{\left(x, \boldsymbol{x}_{\perp}\right)}{\ell}\right) \sim \ell \sigma^{2} .
$$

The Itô-Schrödinger equation (22) is studied in [11], and the statistical moments of (21) can be approximated for a finite $\varepsilon \ll 1$ using the moments of $\hat{u}$, which are derived using Itô's formula. The results are simpler for isotropic statistics, so we henceforth let $\mathcal{R}$ depend on $\left|\boldsymbol{x}_{\perp}\right|$ instead of $\boldsymbol{x}_{\perp}$.

Coherent wave. Conventional imaging modalities neglect the fluctuations of the wave speed and assume the forward model, i.e., the mapping of the source to the wave given by (9) and (12). We now show that the mean wave envelope is not close to $\hat{u}_{o}$, so this forward model is inaccurate and cannot be used even if we had an ensemble of realizations of the medium, which we do not.

Taking the expectation in (22) and recalling equation (10), we obtain the coherent wave

$$
\begin{aligned}
\mathbb{E}\left[\hat{u}\left(x, x_{\perp}, \omega\right)\right] & =\hat{u}_{o}\left(x, x_{\perp}, \omega\right) \exp \left[-\frac{x}{S(\omega)}\right], \\
S(\omega) & =\frac{8}{k^{2}(\omega) \mathcal{R}(0)},
\end{aligned}
$$

which differs from $\hat{u}_{o}$ by the exponential decay on the range scale $S$, the scattering mean free path. We are interested in the scattering regime

$$
L \gg S(\omega),
$$

where the coherent wave is negligible; i.e., the wave is randomized by scattering.

Second moments. The Itô-Schrödinger equation conserves energy, so the decay in (26) means that the random 
fluctuations of $\hat{u}$ gain strength. These fluctuations can be studied using the Wigner transform

$$
\begin{aligned}
W\left(x, \boldsymbol{x}_{\perp}, \boldsymbol{K}, \omega\right)=\int_{\mathbb{R}^{2}} d \tilde{\boldsymbol{x}}_{\perp} e^{-i K \cdot \tilde{\boldsymbol{x}}_{\perp}} \\
\times \hat{u}\left(x, \boldsymbol{x}_{\perp}+\frac{\tilde{\boldsymbol{x}}_{\perp}}{2}, \omega\right) \hat{u}\left(x, \boldsymbol{x}_{\perp}-\frac{\tilde{\boldsymbol{x}}_{\perp}}{2}, \omega\right),
\end{aligned}
$$

where the bar denotes throughout the complex conjugate and $\boldsymbol{K} \in \mathbb{R}^{2}$ is the cross-range component of the wave vector. Note that $W$ is real-valued and satisfies

$$
\int_{\mathbb{R}^{2}} \frac{d \boldsymbol{K}}{(2 \pi)^{2}} W\left(x, \boldsymbol{x}_{\perp}, \boldsymbol{K}, \omega\right)=\left|\hat{u}\left(x, \boldsymbol{x}_{\perp}, \omega\right)\right|^{2},
$$

so we may think of it as a local energy density at $\left(x, x_{\perp}\right)$, resolved over directions in the cross-range plane. However, $W$ may take negative values.

One can obtain from equations (22), (28), and Itô's formula a stochastic evolution equation for $W[13,18]$, starting from the initial condition

$$
\begin{array}{r}
\mathbb{E}\left[W\left(0, \boldsymbol{x}_{\perp}, \boldsymbol{K}, \omega\right)\right]=\left|\hat{f}\left(\omega-\omega_{\mathcal{c}}\right)\right|^{2} 2 \pi r_{0}^{2} \\
\times \exp \left[-\frac{2\left|\boldsymbol{x}_{\perp}\right|^{2}}{r_{0}^{2}}-\frac{|\boldsymbol{K}|^{2} r_{0}^{2}}{2}\right] .
\end{array}
$$

We are particularly interested in the expectation of $W$, which defines the second-order moments of $\hat{u}$ that are relevant for imaging with cross-correlations. This expectation satisfies a radiative transport equation $[2,13]$, which simplifies in the long-range regime (27) to the Fokker-Planck equation [17]

$$
\begin{array}{r}
{\left[\partial_{x}+\frac{\boldsymbol{K} \cdot \nabla_{\boldsymbol{x}_{\perp}}}{k(\omega)}\right] \mathbb{E}\left[W\left(x, \boldsymbol{x}_{\perp}, \boldsymbol{K}, \omega\right)\right]} \\
=k^{2}(\omega) D_{\boldsymbol{K}} \Delta_{\boldsymbol{K}} \mathbb{E}\left[W\left(x, \boldsymbol{x}_{\perp}, \boldsymbol{K}, \omega\right)\right], \\
x>0,
\end{array}
$$

with diffusion coefficient $D_{K}(\omega)=\left|\mathcal{R}^{\prime \prime}(0)\right| / 8$, where $\nabla_{\boldsymbol{x}_{\perp}}$ is the gradient in $\boldsymbol{x}_{\perp}$ and $\Delta_{\boldsymbol{K}}$ is the Laplacian in $\boldsymbol{K}$. The diffusion in $\boldsymbol{K}$ describes the spread of energy over directions due to cumulative scattering.

The Fokker-Plank equation can be solved explicitly by taking the Fourier transform in $\boldsymbol{x}_{\perp}$ and $\boldsymbol{K}$. Using its solution in the expectation of (28), we obtain the second moments

$$
\begin{array}{r}
\mathbb{E}\left[\hat{u}\left(x, \boldsymbol{x}_{\perp}+\frac{\tilde{\boldsymbol{x}}_{\perp}}{2}, \omega\right) \overline{\left.\hat{u}\left(x, \boldsymbol{x}_{\perp}-\frac{\tilde{\boldsymbol{x}}_{\perp}}{2}, \omega\right)\right]}\right. \\
=\left|\hat{f}\left(\omega-\omega_{c}\right)\right|^{2} \frac{r_{0}^{2}}{r^{2}(x)} \exp \left[-\frac{2\left|\boldsymbol{x}_{\perp}\right|^{2}}{r^{2}(x)}\right. \\
\left.-\frac{\left|\tilde{\boldsymbol{x}}_{\perp}\right|^{2}}{\chi^{2}(x, \omega)}+i \phi\right],
\end{array}
$$

where

$$
\begin{aligned}
r(x) & =r_{0}\left(1+\frac{8 D_{K} x^{3}}{3 r_{0}^{2}}\right)^{\frac{1}{2}}, \\
\chi(x, \omega) & =\frac{1}{k(\omega) \sqrt{x D_{K}}}\left(\frac{1+\frac{8 D_{K} x^{3}}{3 r_{0}^{2}}}{1+\frac{2 D_{K} x^{3}}{3 r_{0}^{2}}}\right)^{\frac{1}{2}},
\end{aligned}
$$

and $\phi$ is a phase written explicitly in $[13,14]$.

Let us compare the modulus of the second moments (32) with

$$
\begin{aligned}
& \left|\hat{u}_{o}\left(x, x_{\perp}+\frac{\tilde{x}_{\perp}}{2}, \omega\right) \overline{\hat{u}_{o}\left(x, x_{\perp}-\frac{\tilde{x}_{\perp}}{2}, \omega\right)}\right| \\
& \quad=\left|\hat{f}\left(\omega-\omega_{c}\right)\right|^{2} \exp \left[-\frac{2\left|\boldsymbol{x}_{\perp}\right|^{2}}{r_{0}^{2}}-\frac{\left|\tilde{\boldsymbol{x}}_{\perp}\right|^{2}}{2 r_{0}^{2}}\right],
\end{aligned}
$$

obtained from (12) and the assumption (20). We note that the radius (33) of the beam, the length scale of decay in $\left|\boldsymbol{x}_{\perp}\right|$ in (32), is larger than the radius $r_{0}$ in the homogeneous medium. Cumulative scattering spreads the energy over directions in the cross-range plane, so it widens the beam. The scale $\mathcal{X}$ of decay of (32) in $\left|\tilde{\boldsymbol{x}}_{\perp}\right|$ is called the decoherence length. It models the statistical decorrelation of the wave over cross-range offsets, and its definition (34) shows that it is proportional to the wavelength and decreases with $x$. At the range $x=L$ of the array, we estimate from equations (8), (25), (27), and (33) that the decoherence length is

$$
\chi(L, \omega) \sim \ell \sqrt{S(\omega) / L} \ll \ell \ll r_{0} .
$$

Two-frequency moments. The Wigner transform (28) is useful for analyzing the energy resolved over directions. In imaging we are also interested in the energy resolved over time, so we use the two-frequency Wigner transform

$$
\begin{gathered}
\mathcal{W}\left(x, \boldsymbol{x}_{\perp}, \boldsymbol{K}, \omega, T\right) \\
=\int_{\mathbb{R}} \frac{d \tilde{\omega}}{2 \pi} e^{-i \tilde{\omega} T} \int_{\mathbb{R}^{2}} d \tilde{\boldsymbol{x}}_{\perp} e^{-i \boldsymbol{K} \cdot \tilde{\boldsymbol{x}}_{\perp}} \\
\times \hat{u}\left(x, \boldsymbol{x}_{\perp}+\frac{\tilde{\boldsymbol{x}}_{\perp}}{2}, \omega+\frac{\tilde{\omega}}{2}\right) \hat{\hat{u}\left(x, \boldsymbol{x}_{\perp}-\frac{\widetilde{\boldsymbol{x}}_{\perp}}{2}, \omega-\frac{\tilde{\omega}}{2}\right) .}
\end{gathered}
$$

The two-frequency moments are given by the Fourier transform of $\mathbb{E}[\mathcal{W}]$, calculated in [9]. Their expression is complicated, but in our regime the net effect of the random medium is, in addition to the diffusion over the wave vectors $K$ seen in the previous section, a diffusion in the time variable $T$, with diffusion coefficient $D_{T}=\mathcal{R}(0) /\left(8 c_{o}^{2}\right)$.

The waves decorrelate over frequency offsets, and this manifests as an exponential decay in $|\tilde{\omega}|$ of the second moments on a frequency scale $\Omega$ called the decoherence frequency [7]. At $x=L$, this scale is

$$
\Omega(L) \sim \frac{1}{\sqrt{D_{T} L}}=\omega \sqrt{\frac{S(\omega)}{L}} \ll \omega .
$$


Smoothed Wigner transform. The Wigner transforms (28) and (37) are not self-averaging, but they can be stabilized statistically by convolution with an appropriate kernel $[1,4,18]$.

The fourth-order moments calculated recently in [14] have facilitated the explicit analysis of statistical stability of the smoothed Wigner transform

$$
\begin{aligned}
& W_{s}\left(x, \boldsymbol{x}_{\perp}, \boldsymbol{K}, \omega\right)=\int_{\mathbb{R}^{2}} d \boldsymbol{x}_{\perp}^{\prime} \int_{\mathbb{R}^{2}} d \boldsymbol{K}^{\prime} \\
& \times W\left(x, \boldsymbol{x}_{\perp}-\boldsymbol{x}_{\perp}^{\prime}, \boldsymbol{K}-\boldsymbol{K}^{\prime}, \omega\right) \exp \left[-\frac{\left|\boldsymbol{x}_{\perp}^{\prime}\right|^{2}}{R_{S}^{2}}-\frac{\left|\boldsymbol{K}^{\prime}\right|^{2}}{K_{S}^{2}}\right],
\end{aligned}
$$

which satisfies [14]

$$
\frac{\operatorname{std}\left[\mathrm{W}_{\mathrm{S}}\left(\mathrm{x}, \boldsymbol{x}_{\perp}, \boldsymbol{K}, \omega\right)\right]}{\mathbb{E}\left[W_{S}\left(x, \boldsymbol{x}_{\perp}, \boldsymbol{K}, \omega\right)\right]} \approx \frac{\left[\chi(x, \omega) K_{S}\right]^{2}+4}{\left[\chi(x, \omega) K_{S}\right]^{2}+4\left(R_{S} K_{S}\right)^{2}},
$$

where "std" denotes the standard deviation. This shows that $W_{s}$ can be approximated by its expectation when the smoothing parameters satisfy $R_{s} K_{s} \gg 1$. Similarly, we expect that the two-frequency Wigner transform is stabilized by smoothing [18]. In particular, the smoothing parameter $T_{s}$ in $T$ and the support $B$ in $\omega-\omega_{c}$ should satisfy $B T_{s} \gg 1$.

Imaging the source. The data for imaging the source $S$ are gathered by an array of receivers supported in $\mathcal{A} \subset \mathbb{R}^{2}$ at range $L$,

$$
\mathcal{D}\left(\boldsymbol{x}_{\perp}, t\right)=\left\{P\left(\left(L, x_{\perp}\right), t\right), \quad x_{\perp} \in \mathcal{A}, t \in \mathbb{R}\right\} .
$$

Although imaging can be done without knowing the pulse, we assume for simplicity that we know it and estimate the spatial profile of (3) by the solution of the linear least squares problem

$$
\rho_{S}^{\mathrm{LS}}=\operatorname{argmin}_{\rho_{S} \in L^{2}\left(\mathbb{R}^{3}\right)}\left\|\mathcal{D}-\mathcal{L} \rho_{S}\right\|_{L^{2}(\mathcal{A} \times \mathbb{R})}^{2} .
$$

Here $\mathcal{L}: L^{2}\left(\mathbb{R}^{3}\right) \mapsto L^{2}(\mathcal{A} \times \mathbb{R})$ is the forward map $\rho_{s} \mapsto$ $\mathcal{D}$ defined for the guess (search) source

$$
S(t)=f(t) e^{-i \omega_{c} t} \rho_{S}(\overrightarrow{\boldsymbol{x}}),
$$

using (21) and linear superposition

$$
\begin{array}{r}
\mathcal{L} \rho_{S}\left(\boldsymbol{x}_{\perp}, t\right)=\int_{\mathbb{R}} \frac{d \omega}{2 \pi} e^{-i \omega t} \hat{f}\left(\omega-\omega_{c}\right) \\
\times \int_{\mathbb{R}^{3}} d \boldsymbol{y} \hat{G}\left(\left(L, \boldsymbol{x}_{\perp}\right), \overrightarrow{\boldsymbol{y}}, \omega\right) \rho_{S}(\overrightarrow{\boldsymbol{y}}), \\
\boldsymbol{x}_{\perp} \in \mathcal{A}, t \in \mathbb{R},
\end{array}
$$

where $\hat{G}$ is the Green's function of the Helmholtz equation in the paraxial regime.

The minimizer in (42) satisfies the normal equations

$$
\mathcal{L}^{\star} \mathcal{L} \rho_{S}^{\mathrm{LS}}(\overrightarrow{\boldsymbol{y}})=\mathcal{L}^{\star} \mathcal{D}(\overrightarrow{\boldsymbol{y}}), \quad \overrightarrow{\boldsymbol{y}}=\left(y, \boldsymbol{y}_{\perp}\right) \in \mathbb{R}^{3},
$$

where $\mathcal{L}^{\star}$ is the adjoint of $\mathcal{L}$,

$$
\begin{aligned}
& \mathcal{L}^{\star} \mathcal{D}(\overrightarrow{\boldsymbol{y}})=\int_{\mathbb{R}} \frac{d \omega}{2 \pi} \overline{\hat{f}\left(\omega-\omega_{c}\right)} \\
& \times \int_{\mathcal{A}} d \boldsymbol{x}_{\perp} \overline{\hat{G}}\left(\left(L, \boldsymbol{x}_{\perp}\right), \overrightarrow{\boldsymbol{y}}, \omega\right) \widehat{\mathcal{D}}\left(\boldsymbol{x}_{\perp}, \omega\right) .
\end{aligned}
$$

The normal operator $\mathcal{L}^{\star} \mathcal{L}$ is rarely computed in imaging and it is not invertible, so problem (42) needs regularization. However, a closer look reveals that

$$
\mathcal{L}^{\star} \mathcal{L} \rho_{S}(\overrightarrow{\boldsymbol{y}})=\int_{\mathbb{R}^{3}} d \overrightarrow{\boldsymbol{y}}^{\prime} \mathcal{K}\left(\overrightarrow{\boldsymbol{y}}, \overrightarrow{\boldsymbol{y}}^{\prime}\right) \rho_{S}\left(\overrightarrow{\boldsymbol{y}}^{\prime}\right)
$$

has the kernel

$$
\begin{aligned}
& \mathcal{K}\left(\overrightarrow{\boldsymbol{y}}, \overrightarrow{\boldsymbol{y}}^{\prime}\right)=\int_{\mathbb{R}} \frac{d \omega}{2 \pi}\left|\hat{f}\left(\omega-\omega_{\mathcal{C}}\right)\right|^{2} \\
& \quad \times \int_{\mathcal{A}} d \boldsymbol{x}_{\perp} \hat{G}\left(\left(L, \boldsymbol{x}_{\perp}\right), \overrightarrow{\boldsymbol{y}}^{\prime}, \omega\right) \overline{\hat{G}\left(\left(L, \boldsymbol{x}_{\perp}\right), \overrightarrow{\boldsymbol{y}}, \omega\right),}
\end{aligned}
$$

which is peaked on the diagonal. This kernel is the mathematical model of a famous experiment called time reversal [10], where the wave from a point source at $\overrightarrow{\boldsymbol{y}}$, emitting a signal with Fourier transform $|\widehat{f}|^{2}$, is received at the array in $\mathcal{A}$, it is time reversed (hence the complex conjugation in (48)), and it is sent back in the medium, where it is observed at point $\overrightarrow{\boldsymbol{y}}^{\prime}$. We expect from the time reversibility of the wave equation that $\mathcal{K}$ should be peaked on the diagonal if the array captures most of the wave. This is indeed what happens in homogeneous media. The amazing fact demonstrated in [10] and analyzed in our regime in [18] is that in random media $\mathcal{K}$ is highly peaked even if the array has small aperture. Even more striking, $\mathcal{K}$ is self-averaging The implication is that we can replace the normal operator $\mathcal{L}^{\star} \mathcal{L}$ in (45) by the identity and obtain the time reversal function

$$
\mathcal{J}_{\mathrm{TR}}(\overrightarrow{\boldsymbol{y}})=\mathcal{L}^{\star} \mathcal{D}(\overrightarrow{\boldsymbol{y}}), \quad \overrightarrow{\boldsymbol{y}} \in \mathbb{R}^{3} .
$$

This does not give a quantitative estimate of the source, but it peaks at points $\overrightarrow{\boldsymbol{y}}$ in its spatial support.

Reverse time migration. As nice as (49) is in theory, it cannot be calculated using (46) because the medium is not known. Conventional imaging calculates instead

$$
\begin{aligned}
& \mathcal{J}_{\text {mig }}(\overrightarrow{\boldsymbol{y}})=\mathcal{L}_{0}^{\star} \mathcal{D}(\overrightarrow{\boldsymbol{y}})=\int_{\mathbb{R}} \frac{d \omega}{2 \pi} \overline{\hat{f}} \overline{\left(\omega-\omega_{c}\right)} \\
& \times \int_{\mathcal{A}} d \boldsymbol{x}_{\perp} \overline{\hat{G}_{o}\left(\left(L, \boldsymbol{x}_{\perp}\right), \overrightarrow{\boldsymbol{y}}, \omega\right)} \widehat{\mathcal{D}}\left(\boldsymbol{x}_{\perp}, \omega\right), \quad \overrightarrow{\boldsymbol{y}}=\left(y, \boldsymbol{y}_{\perp}\right),
\end{aligned}
$$

where the unknown $\hat{G}$ in (46) is replaced by the Green's function $\hat{G}_{o}$ of the Helmholtz equation with constant wave speed $c_{o}$,

$$
\hat{G}_{o}\left(\left(L, \boldsymbol{x}_{\perp}\right), \overrightarrow{\boldsymbol{y}}, \omega\right) \approx \frac{\exp \left[i \omega \tau\left(\left(L, \boldsymbol{x}_{\perp}\right), \overrightarrow{\boldsymbol{y}}\right)\right]}{4 \pi(L-y)} .
$$


Here the approximation is for $L-y \gg\left|\boldsymbol{y}_{\perp}\right|,\left|\boldsymbol{x}_{\perp}\right|$, and $\boldsymbol{\tau}$ is the travel time in the paraxial regime

$$
\begin{aligned}
\boldsymbol{\tau}\left(\left(L, \boldsymbol{x}_{\perp}\right), \overrightarrow{\boldsymbol{y}}\right) & =\frac{1}{c_{o}}\left[L-y+\frac{\left|\boldsymbol{x}_{\perp}-\boldsymbol{y}_{\perp}\right|^{2}}{2(L-y)}\right] \\
& \approx \frac{\left|\left(L, \boldsymbol{x}_{\perp}\right)-\overrightarrow{\boldsymbol{y}}\right|}{c_{o}} .
\end{aligned}
$$

The role of $\hat{G}_{o}$ in (50) is described as "back-propagation" or "migration" to $\vec{y}$ of the wave received at the array, and $\mathcal{J}_{\mathrm{mig}}$ is called the "reverse time migration" image. In our regime it amounts to convolving the data with the time-reversed pulse

$$
\mathcal{D}_{f}\left(\boldsymbol{x}_{\perp}, t\right)=\left[f(-t) e^{i \omega_{c} t}\right] \star_{t} \mathcal{D}\left(\boldsymbol{x}_{\perp}, t\right),
$$

superposing it over the aperture and evaluating the result at $t=\tau$ :

$$
\mathcal{J}_{\text {mig }}(\vec{y}) \approx \frac{1}{4 \pi(L-y)} \int_{\mathcal{A}} d x_{\perp} \mathcal{D}_{f}\left(x_{\perp}, \tau\left(\left(L, x_{\perp}\right), \vec{y}\right)\right) .
$$

The geometrical factor in front of the integral is usually neglected in imaging.

The reverse-time migration method is used in many imaging applications and gives good results if the coherent wave reaching the array is strong. However, we know from the section "Coherent wave" that in our regime this wave is negligible, so the expectation of $\mathcal{J}_{\text {mig }}$ is negligible. Moreover, the standard deviation of $\mathcal{J}_{\text {mig, which is deter- }}$ mined by the second moments analyzed above, is finite. This implies that $\mathcal{J}_{\text {mig }}$ is not a good image because it has large random fluctuations. In practice, these manifest as spurious peaks (see middle plot in Figure 5) that change unpredictably with the realization of the medium.

Coherent interferometric imaging. Consider the empirical cross-correlations of $\mathcal{D}_{f}$, back-propagated to $\overrightarrow{\boldsymbol{y}}$ using the travel time (52), and calculated in a time window $\Phi$ of duration of order $1 / \widetilde{\Omega}$. We write them here using the Fourier transform in $t$,

$$
\begin{aligned}
& C_{\overrightarrow{\boldsymbol{y}}}\left(\boldsymbol{x}_{\perp}, \boldsymbol{x}_{\perp}^{\prime} ; \tilde{\Omega}\right) \\
& =\int_{\mathbb{R}} \frac{d \omega}{2 \pi} \int_{\mathbb{R}} \frac{d \omega^{\prime}}{2 \pi} \hat{\Phi}\left(\frac{\omega-\omega^{\prime}}{\widetilde{\Omega}}\right) \overline{\hat{f}\left(\omega-\omega_{\mathcal{C}}\right)} \\
& \quad \times \hat{f}\left(\omega^{\prime}-\omega_{\mathcal{C}}\right) \widehat{\mathcal{D}}\left(\boldsymbol{x}_{\perp}, \omega\right) \overline{\widehat{\mathcal{D}}\left(\boldsymbol{x}_{\perp}^{\prime}, \omega^{\prime}\right)} \\
& \times \exp \left[-i \omega \tau\left(\left(L, \boldsymbol{x}_{\perp}\right), \overrightarrow{\boldsymbol{y}}\right)+i \omega^{\prime} \tau\left(\left(L, \boldsymbol{x}_{\perp}^{\prime}\right), \overrightarrow{\boldsymbol{y}}\right)\right],
\end{aligned}
$$

where $\hat{\Phi}$ is the Fourier transform of the window function $\Phi$. As explained in "Two frequency moments," the wave components decorrelate statistically over frequency offsets, so in (55) we use $\widehat{\Phi}$ to keep $\left|\omega-\omega^{\prime}\right| \leq \widetilde{\Omega}$. We also know that the wave decorrelates over cross-range offsets, so we need another window $\Psi$ to enforce $\left|\boldsymbol{x}_{\perp}-\boldsymbol{x}_{\perp}^{\prime}\right| \leq \tilde{\mathcal{X}}$. The coherent interferometric (CINT) imaging function is

$$
\begin{aligned}
J_{\mathrm{CINT}}(\vec{y} ; \tilde{\chi}, \tilde{\Omega})=\int_{\mathcal{A}} d x_{\perp} & \int_{\mathcal{A}} d x_{\perp}^{\prime} \Psi\left(\frac{\left.\boldsymbol{x}_{\perp}^{\prime}-\boldsymbol{x}_{\perp}\right)}{\tilde{\chi}}\right) \\
& \times C_{\overrightarrow{\boldsymbol{y}}}\left(\boldsymbol{x}_{\perp}, \boldsymbol{x}_{\perp}^{\prime} ; \tilde{\Omega}\right),
\end{aligned}
$$

where the ideal threshold parameters are given by the decoherence length $^{3} \tilde{\chi}=\mathcal{X}(L, \omega)$ and decoherence frequency $\widetilde{\Omega}=\Omega(L)$ given in (34) and (38). Note that these expressions of the decoherence parameters are model specific, so in practice it is best to estimate $\mathcal{X}(L, \omega)$ and $\Omega(L)$ by either some statistical signal processing of the data or adaptively during the formation of the image. The latter was proposed in [7] and amounts to computing (56) with guess values of $\tilde{X}$ and $\widetilde{\Omega}$ that can be adjusted by optimization of some measure of quality of the image.

The window shapes $\Phi$ and $\Psi$ can be arbitrary, but in order to write an explicit expression of the CINT image, we choose them as Gaussians. We also use the Gaussian apodization

$$
\int_{\mathcal{A}} d x_{\perp} \rightsquigarrow \int_{\mathbb{R}^{2}} d x_{\perp} \exp \left(-\left|x_{\perp}\right|^{2} / a^{2}\right)
$$

to avoid dealing with integrals over the bounded set $\mathcal{A}$. With these choices, using the pulse shape (4) and recalling the definition (37) of the two-frequency Wigner transform, we obtain after straightforward calculations that the CINT image is

$$
\begin{aligned}
& \mathcal{J}_{\mathrm{CINT}}(\overrightarrow{\boldsymbol{y}} ; \tilde{\boldsymbol{X}}, \tilde{\Omega}) \propto \int_{\mathbb{R}^{2}} d \boldsymbol{x}_{\perp} \int_{\mathbb{R}^{2}} d \boldsymbol{K} \int_{\mathbb{R}} d \omega \int_{\mathbb{R}} d T \\
& \mathcal{W}\left(L, \boldsymbol{x}_{\perp}, k(\omega) \frac{\left(\boldsymbol{x}_{\perp}-\boldsymbol{y}_{\perp}\right)}{(L-y)}-\boldsymbol{K}, \omega, \frac{\left|\boldsymbol{x}_{\perp}-\boldsymbol{y}_{\perp}\right|^{2}}{2 c_{o}(L-y)}-T\right) \\
& \times \exp \left[-\frac{\left|\boldsymbol{x}_{\perp}\right|^{2}}{a^{2}}-\frac{\left(\omega-\omega_{c}\right)^{2}}{B^{2}}-\frac{|\boldsymbol{K}|^{2}}{K_{s}^{2}}-\frac{T^{2}}{T_{s}^{2}}\right] .
\end{aligned}
$$

Here the symbol $\propto$ denotes equal, up to a constant independent of $\overrightarrow{\boldsymbol{y}}$, and

$$
K_{s}=\frac{2}{\widetilde{\chi}}, \quad T_{s}=\frac{2}{\widetilde{\Omega}}\left(1+\frac{\widetilde{\Omega}^{2}}{4 B^{2}}\right)^{\frac{1}{2}} .
$$

We obtained that the CINT image is given by the smoothed Wigner transform, and the results in the section "Smoothed Wigner transform" imply that it is statistically stable when $a K_{s} \gg 1$ and $B T_{s} \gg 1$. Note that for the optimal choice of the threshold parameters $\tilde{\chi}=\chi(L, \omega)$ and $\widetilde{\Omega}=\Omega(L)$, we have statistical stability when $a \gg \mathcal{X}(L, \omega)$ and $B \gg \Omega(L)$. This result is intuitive, because in (57) we integrate over many decorrelation intervals, and the statistical stabilization is essentially by the law of large numbers.

\footnotetext{
${ }^{3}$ The expression (56) is for a narrowband source. In the broadband case $\tilde{\chi}$ should vary with the frequency, and the expression of $\mathcal{J}_{\mathrm{CINT}}$ is slightly more complicated [7].
} 

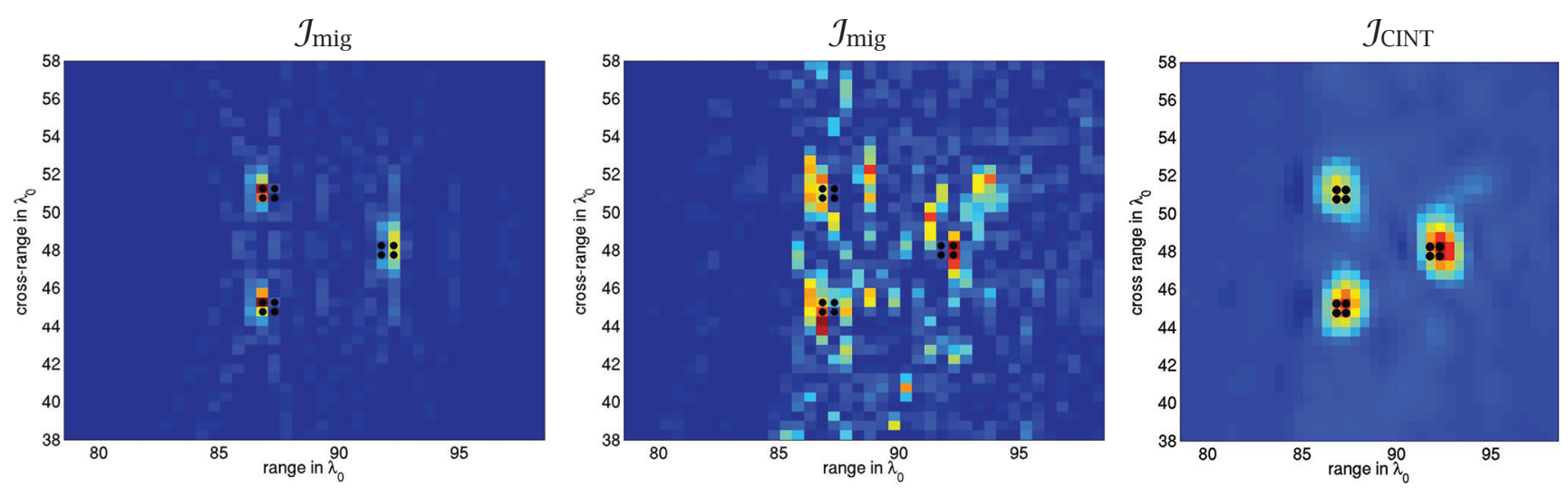

Figure 5. Two-dimensional cross-section of images of three reflectors (black dots) in a homogeneous medium (left) and a medium with microstructure (middle and right). Abscissa and ordinate are range and cross-range in units of $\lambda_{c}$. See Acknowledgments section.

When the statistical stability conditions hold, we can approximate (57) by its expectation and use the second moment formulas described above to analyze the focusing and resolution of the image. This analysis is done in $[4,7]$ and it says that the image peaks in the spatial support of the source (see right plot in Figure 5) and that there is a trade-off between the statistical stability and resolution of the image. The smaller the threshold parameters $\tilde{\chi}$ and $\tilde{\Omega}$, the larger $K_{s}$ and $T_{s}$ and the better the stability. However, the resolution formulas are inverse proportional to $\tilde{X}$ and $\widetilde{\Omega}$, so when these are too small, the images are quite blurry due to oversmoothing in (57).

Inverse scattering. When imaging with an active array (e.g., as in Figure 1) the source $S$ is known because it is in the array. The inverse scattering problem is to determine reflectors in the medium, modeled by a reflectivity $\rho$ added to the coefficient $c^{-2}$ in (1). Writing the wave equation in the form

$$
\begin{aligned}
\frac{1}{c^{2}(\overrightarrow{\boldsymbol{x}})} \partial_{t}^{2} P(\overrightarrow{\boldsymbol{x}}, t)-\Delta_{\overrightarrow{\boldsymbol{x}}} P(\overrightarrow{\boldsymbol{x}}, t) & =S(\overrightarrow{\boldsymbol{x}}, t)-\rho(\overrightarrow{\boldsymbol{x}}) \partial_{t}^{2} P(\overrightarrow{\boldsymbol{x}}, t), \\
t & \in \mathbb{R}, \overrightarrow{\boldsymbol{x}} \in \mathbb{R}^{3},
\end{aligned}
$$

we can view the $\rho$ term as an unknown fictitious source and connect to the inverse source problem discussed above. However, there are two complications: First, the mapping $\rho \mapsto \mathcal{D}$ is nonlinear, because the fictitious source signal depends on the wave $P$. Many imaging modalities neglect this nonlinearity and replace $P$ in the right-hand side of (59) by the wave in the empty medium, without the reflectivity. This is known as the single scattering or Born approximation [8, Chapter 13], and its accuracy is not well understood in random media. Second, the fictitious source signal depends on the wave propagated forward from the array to the reflectors, and after scattering at $\rho$ this wave propagates backward to the array in the same realization of the random medium. The forward and backward going waves are strongly correlated, and this makes the analysis of the imaging function very complicated. One needs to use stochastic invariant imbedding, which introduces transmission and reflection operators in the random medium, as explained in [13].

Nevertheless, CINT imaging has been used successfully for inverse scattering in random media [4,7]. The results displayed in Figure 5 show images of the three reflectors in Figure 3, using the data displayed there. The reverse time migration image is excellent in the medium without fluctuations, but in the random medium it has numerous spurious peaks. The CINT image is nicely focused at the reflectors, although its resolution is worse than that of migration in the homogeneous medium, due to the smoothing for statistical stability. It is possible to improve the resolution using deblurring or other tricks, as discussed for example in [6].

\section{Imaging with Noisy Sources}

Starting from the early 2000s, it has become clear that imaging can be done not only with controlled impulsive sources, which are difficult to come by in some applications (e.g., seismic imaging), but also with random sources like ambient noise. Cross-correlations are essential in dealing with such sources, as explained in depth in [12] and the references therein. We describe briefly this topic and the related virtual source imaging modality $[12$, Chapter $10]$ in random media.

Velocity estimation using noisy sources. In this section only, let the wave speed $c$ in (1) be a smooth and slowly varying unknown function, and suppose that $S$ is a noise source, stationary in time and uncorrelated in space, satisfying

$$
\begin{gathered}
\langle S(\overrightarrow{\boldsymbol{x}}, t)\rangle=0, \\
\left\langle S(\overrightarrow{\boldsymbol{x}}, t) S\left(\overrightarrow{\boldsymbol{x}}^{\prime}, t^{\prime}\right)\right\rangle=\rho_{S}(\overrightarrow{\boldsymbol{x}}) \delta\left(\overrightarrow{\boldsymbol{x}}-\overrightarrow{\boldsymbol{x}}^{\prime}\right) f\left(t-t^{\prime}\right) .
\end{gathered}
$$

Here $\langle\cdot\rangle$ denotes the expectation with respect to the distribution of $S, \rho_{S}$ models the strength and compact spatial 
support of the source, and $f$ is the time correlation.

The wave generated by $S$ is measured by receiver sensors at locations $\overrightarrow{\boldsymbol{x}}_{r}$, for $r=1, \ldots, N$. These locations may be nearby or widely spaced. As in the previous section, denote by $\mathcal{D}$ the measurements (data). Their model is

$$
\begin{array}{r}
\mathcal{D}\left(\overrightarrow{\boldsymbol{x}}_{r}, t\right)=\int_{\mathbb{R}} d t^{\prime} \int_{\mathbb{R}^{3}} d \overrightarrow{\boldsymbol{y}} S\left(\overrightarrow{\boldsymbol{y}}, t-t^{\prime}\right) G\left(\overrightarrow{\boldsymbol{x}}_{r}, \overrightarrow{\boldsymbol{y}}, t^{\prime}\right), \\
r=1, \ldots, N_{r}, t \in[0, T],
\end{array}
$$

where $G$ is the causal Green's function of equation (1). These data look like noise, but they carry information about the unknown medium. When the duration $T$ of the measurements is long enough, this information can be extracted from the empirical cross-correlations

$$
\begin{gathered}
C_{T}\left(\overrightarrow{\boldsymbol{x}}_{r}, \overrightarrow{\boldsymbol{x}}_{r^{\prime}}, t\right)=\frac{1}{T} \int_{0}^{T} d t^{\prime} \mathcal{D}\left(\overrightarrow{\boldsymbol{x}}_{r}, t^{\prime}\right) \mathcal{D}\left(\overrightarrow{\boldsymbol{x}}_{r^{\prime}}, t^{\prime}+t\right) \\
r, r^{\prime}=1, \ldots, N
\end{gathered}
$$

which converge as $T \rightarrow \infty$, in probability, to the statistical cross-correlations [12, Chapter 2]

$$
\begin{aligned}
& C_{T}\left(\overrightarrow{\boldsymbol{x}}_{r}, \overrightarrow{\boldsymbol{x}}_{r^{\prime}}, t\right) \stackrel{T \rightarrow \infty}{\longrightarrow} C\left(\overrightarrow{\boldsymbol{x}}_{r}, \overrightarrow{\boldsymbol{x}}_{r^{\prime}}, t\right) \\
& \quad=\int_{\mathbb{R}} d t^{\prime} \int_{\mathbb{R}} d t^{\prime \prime} f\left(t+t^{\prime}-t^{\prime \prime}\right) \\
& \quad \times \int_{\mathbb{R}^{3}} d \overrightarrow{\boldsymbol{y}} \rho_{s}(\overrightarrow{\boldsymbol{y}}) G\left(\overrightarrow{\boldsymbol{x}}_{r}, \overrightarrow{\boldsymbol{y}}, t^{\prime}\right) G\left(\overrightarrow{\boldsymbol{x}}_{r^{\prime}}, \overrightarrow{\boldsymbol{y}}, t^{\prime \prime}\right) .
\end{aligned}
$$

The result (63) is used in [12, Chapter 2] and the references therein for a variety of source configurations. A particularly favorable one corresponds to a source density $\rho_{s}$ supported on a large sphere surrounding all the receivers. With such $\rho_{s}$ and assuming that the wave speed equals the constant $c_{o}$ outside this sphere, it follows from the Helmholtz-Kirchhoff identity ${ }^{4}$ [12, Section 2.1.3] that

$$
\partial_{t} C\left(\overrightarrow{\boldsymbol{x}}_{r}, \overrightarrow{\boldsymbol{x}}_{r^{\prime}}, t\right)=-\frac{\mathcal{C}_{o}}{2}\left[G_{f}\left(\overrightarrow{\boldsymbol{x}}_{r}, \overrightarrow{\boldsymbol{x}}_{r^{\prime}}, t\right)-G_{f}\left(\overrightarrow{\boldsymbol{x}}_{r}, \overrightarrow{\boldsymbol{x}}_{r^{\prime}},-t\right)\right],
$$

where

$$
G_{f}\left(\overrightarrow{\boldsymbol{x}}_{r}, \overrightarrow{\boldsymbol{x}}_{r^{\prime}}, t\right)=f(t) \star_{t} G\left(\overrightarrow{\boldsymbol{x}}_{r}, \overrightarrow{\boldsymbol{x}}_{r^{\prime}}, t\right),
$$

where we recall that $\star_{t}$ denotes convolution in time. Thus, the cross-correlations of the noise-like data (61) give the smoothed Green's function at $\overrightarrow{\boldsymbol{x}}_{r}$, corresponding to a virtual source at $\overrightarrow{\boldsymbol{x}}_{r^{\prime}}$.

To showcase this remarkable result, consider the geometrical optics approximation of the Green's function, which holds for sufficiently slowly varying wave speed $c$. We have

$$
\begin{gathered}
G\left(\overrightarrow{\boldsymbol{x}}_{r}, \overrightarrow{\boldsymbol{x}}_{r^{\prime}}, t\right) \approx \alpha\left(\overrightarrow{\boldsymbol{x}}_{r}, \overrightarrow{\boldsymbol{x}}_{r^{\prime}}\right) \delta\left(t-\tau\left(\overrightarrow{\boldsymbol{x}}_{r}, \overrightarrow{\boldsymbol{x}}_{r^{\prime}}\right)\right), \\
\boldsymbol{\tau}\left(\overrightarrow{\boldsymbol{x}}_{r}, \overrightarrow{\boldsymbol{x}}_{r^{\prime}}\right)=\int_{\Gamma\left(\overrightarrow{\boldsymbol{x}}_{r}, \overrightarrow{\boldsymbol{x}}_{r^{\prime}}\right)} \frac{d s}{C(\overrightarrow{\boldsymbol{x}}(s))},
\end{gathered}
$$

\footnotetext{
${ }^{4}$ This is an application of Green's second identity for the Helmholtz equation and the Sommerfeld radiation condition.
}

where $\boldsymbol{\tau}\left(\overrightarrow{\boldsymbol{x}}_{r}, \overrightarrow{\boldsymbol{x}}_{s}\right)$ is the travel time from $\overrightarrow{\boldsymbol{x}}_{r^{\prime}}$ to $\overrightarrow{\boldsymbol{x}}_{r}$, given by the integral of $1 / \mathrm{C}$ along the ray $\Gamma$ (characteristic of the eikonal equation) with arc length denoted by $S$, and $\alpha$ denoting the amplitude factor that accounts for geometrical spreading. Since $f(t)$ is peaked at $t=0$, we obtain from (64) that the cross-correlation of the data displays two peaks, at $t= \pm \tau\left(\overrightarrow{\boldsymbol{x}}_{r}, \overrightarrow{\boldsymbol{x}}_{r^{\prime}}\right)$. Thus, the crosscorrelations allow the estimation of travel times, which can then be used for the inverse tomographic problem of estimating the wave speed $c$.

The idea of using ambient noise to obtain travel times has been proposed for helioseismology and exploration seismology since the late 1960s. The noise sources come, for example, from the interaction of ocean waves with the ocean bottom and the coast. These generate surface waves, and geophysicsts have used equations like (64) to carry out tomographic velocity estimations in the Southern California region, in Tibet, and in the Alps.

Virtual source imaging. The results in the previous section extend to the problem of imaging reflectors in a medium with known and slowly varying wave speed, using a passive array of receiver sensors [12, Chapter 5] that measure the wave due to noisy sources. A good source configuration for this problem is known as "daylight illumination" [12, Section 5.3], where the noise sources lie on one side of the array and the reflectors are on the opposite side.

Say for simplicity that there is a single, point-like reflector at location $\overrightarrow{\boldsymbol{y}}_{\star}$, which is at large distance from the passive array compared to the aperture of the array in $\mathcal{A}$. Then, with the daylight illumination, the cross-correlations (62) have four peaks, at time

$$
t= \pm \boldsymbol{\tau}\left(\overrightarrow{\boldsymbol{x}}_{r}, \overrightarrow{\boldsymbol{x}}_{r^{\prime}}\right), \quad t= \pm\left[\boldsymbol{\tau}\left(\overrightarrow{\boldsymbol{y}}_{\star}, \overrightarrow{\boldsymbol{x}}_{r^{\prime}}\right)+\boldsymbol{\tau}\left(\overrightarrow{\boldsymbol{x}}_{r}, \overrightarrow{\boldsymbol{y}}_{\star}\right)\right]
$$

Because the first two peaks are near $t \approx 0$, they can be muted by time windowing the cross-correlations, and the result can be used to form the reverse time migration type image (recall (54))

$$
\begin{aligned}
& \mathcal{I}_{\text {daylight }}(\overrightarrow{\boldsymbol{y}})=\int_{\mathcal{A}} d s_{\overrightarrow{\boldsymbol{x}}} \int_{\mathcal{A}} d s_{\overrightarrow{\boldsymbol{x}}^{\prime}} \\
& \quad \times C_{T}\left(\overrightarrow{\boldsymbol{x}}, \overrightarrow{\boldsymbol{x}}^{\prime}, \boldsymbol{\tau}\left(\overrightarrow{\boldsymbol{y}}, \overrightarrow{\boldsymbol{x}}^{\prime}\right)+\boldsymbol{\tau}(\overrightarrow{\boldsymbol{x}}, \overrightarrow{\boldsymbol{y}})\right) .
\end{aligned}
$$

This image is large in the vicinity of $\overrightarrow{\boldsymbol{y}}_{\star}$, because there we superpose the cross-correlations at their peak.

The virtual source imaging modality [12, Chapters 9,10] is similar to daylight imaging, but there are no noise sources. Instead, the illumination comes from an array of controlled sources, and the wave passes through a strongly scattering medium before it is measured at the receiver sensors. An interesting application of this modality is the synthetic aperture imaging of fast-moving objects (satellites or energetic debris) orbiting the earth, where the sources are powerful antennas on the ground and the receivers are mounted on aircraft that fly over the turbulent atmosphere 


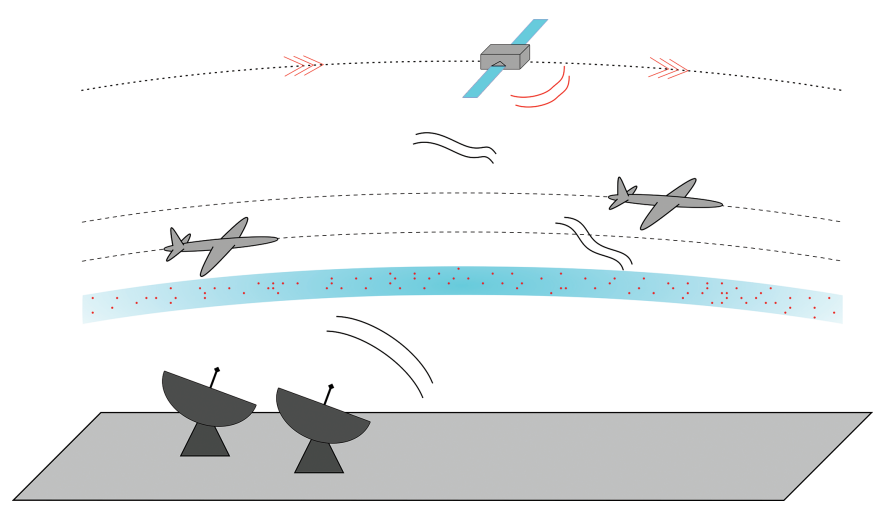

Figure 6. Illustration of a virtual source setup for imaging objects orbiting the earth.

[3], as illustrated in Figure 6. We refer the reader to [12, Chapter 10] for the analysis of the virtual source imaging method using the paraxial theory of wave propagation in random media summarized in the section "Gaussian beam in the random medium" and to [3] for the analysis of the imaging modality illustrated in Figure 6.

\section{Final Remarks}

The presentation in this article has focused mostly on the paraxial regime in media with strongly mixing properties of the fluctuations of the wave speed. Extensions of the wave propagation theory to random media with long-range properties have been obtained recently, and they show that under certain scaling assumptions, the wave can be described by a fractional Itô-Schrödinger equation. Some progress has also been made in the analysis of beam propagation in complicated random media like the turbulent atmosphere, using the Kolmogorov scaling model for the velocity fluctuations. Still, random media that cannot be analyzed using stochastic asymptotic limits based on assumptions of separation of scales remain poorly understood.

Time dependence of random media has been largely ignored because of technical difficulties. The usual argument for this neglect is that media change on a much longer time scale than the duration of propagation of the waves from the sources to the receivers. This is mostly true for electromagnetic waves, but sound waves may be affected by motion, especially in narrowband regimes. The recent study [5] analyzes beam propagation in slowly moving random media and shows that the motion has at least two beneficial effects for imaging: First, cumulative scattering causes transfer of energy over the frequencies and thus widens the bandwidth of the probing signals. This in turn improves the range resolution of images. Second, time changes give access to different realizations of the random medium which can be used to obtain selfaveraging estimates of the Wigner transform, which is the main tool for coherent imaging in random media.
In this article we considered coherent imaging modalities, where both the amplitude and phase of the wave are measured. It is not always possible to measure the phase, and cumulative scattering in the random medium may be so strong that the phase information is not useful. Thus, imaging must be carried out with measurements of the intensity of the wave. It turns out that there is a lot of information in the speckle patterns (fluctuations) of the intensity [12, Chapter 11], and experimentalists have been clever in extracting it. New imaging modalities are emerging, and mathematicians can play a key role in further development of these methods, placing them in a rigorous framework and endowing them with resolution theories that take into account the uncertainty of the medium as well as unconventional sources of waves.

ACKNOWLEDGMENTS. The author thanks Jörn Zimmerling for the illustrations in Figures 1, 2, 4, and 6.

She also thanks Ricardo Alonso, Josselin Garnier, Ilker Kocyigit, George Papanicolaou, Knut Sølna, and Chrysoula Tsogka for the collaboration on the topics in this article.

Current and past support from AFOSR (program manager Dr. Arje Nachman), NSF, and ONR (program manager Dr. Reza Malek-Madani) is gratefully acknowledged.

Due to restrictions on the allowed number of references, the author could not acknowledge many important contributions in the field of imaging in random media. The reader is invited to consult the bibliography in the cited references.

\section{References}

[1] Bal G, Komorowski T, Ryzhik L. Self-averaging of Wigner transforms in random media. Communications in Mathematical Physics, 242(1-2):81-135, 2003. MR2018270

[2] Borcea L, Garnier J. Derivation of a one-way radiative transfer equation in random media. Physical Review E, 93(2):022115, 2016.

[3] Borcea L, Garnier J, Papanicolaou GC, Sølna K, Tsogka C. Resolution analysis of passive synthetic aperture imaging of fast moving objects. SIAM Journal on Imaging Sciences, 10(2):665-710, 2017. MR3650424

[4] Borcea L, Garnier J, Papanicolaou GC, Tsogka C. Enhanced statistical stability in coherent interferometric imaging. Inverse problems, 27(8):085004, 2011. MR2819946

[5] Borcea L, Garnier J, Sølna K. Wave propagation and imaging in moving random media. Multiscale Modeling \& Simulation, 17(1):31-67, 2019. MR3895329

[6] Borcea L, Kocyigit I. Passive array imaging in random media. IEEE Transactions on Computational Imaging, 4(3):459469, 2018. MR3847787

[7] Borcea L, Papanicolaou GC, Tsogka C. Adaptive interfero- 
metric imaging in clutter and optimal illumination. Inverse Problems, 22(4):1405, 2006. MR2249471

[8] Born M, Wolf E. Principles of optics: Electromagnetic theory of propagation, interference and diffraction of light. Cambridge University Press, 7th edition, 1999. MR0108202

[9] Fannjiang AC. White-noise and geometrical optics limits of Wigner-Moyal equation for beam waves in turbulent media II: Two-frequency formulation. Journal of Statistical Physics, 120(3-4):543-586, 2005. MR2182320

[10] Fink M, Cassereau D, Derode A, Prada C, Roux P, Tanter M, Thomas J-L, Wu F. Time-reversed acoustics. Reports on Progress in Physics, 63(12):1933, 2000.

[11] Fouque J-P, Papanicolaou GC, Samuelides Y. Forward and Markov approximation: the strong-intensityfluctuations regime revisited. Waves in Random Media, 8(3):303-314, 1998. MR1633157

[12] Garnier J, Papanicolaou GC. Passive imaging with ambient noise. Cambridge University Press, 2016. MR3616275

[13] Garnier J, Sølna K. Coupled paraxial wave equations in random media in the white-noise regime. Annals of Applied Probability, 19(1):318-346, 2009. MR2498680

[14] Garnier J, Sølna K. Fourth-moment analysis for wave propagation in the white-noise paraxial regime. Archive for Rational Mechanics and Analysis, 220(1):37-81, 2016. MR3458158

[15] Ishimaru A. Wave propagation and scattering in random media, Volume 2. Academic Press, New York, 1978. MR1626707

[16] Keller JB. Stochastic Processes in Mathematical Physics and Engineering, Volume 16 of Proceedings of Symposia in Applied Mathematics. AMS, 1964.

[17] Kesten H, Papanicolaou GC. A limit theorem for stochastic acceleration. Communications in Mathematical Physics, 78(1):19-63, 1980. MR597029

[18] Papanicolaou GC, Ryzhik L, Sølna K. Self-averaging from lateral diversity in the Itô-Schrödinger equation. Multiscale Modeling \& Simulation, 6(2):468-492, 2007. MR2338491

[19] Ryzhik L, Papanicolaou GC, Keller JB. Transport equations for elastic and other waves in random media. Wave Motion, 24(4):327-370, 1996. MR1427483

[20] Tatarski VI. Wave propagation in a turbulent medium. McGraw-Hill Book Company, New York, 1961. MR0127671

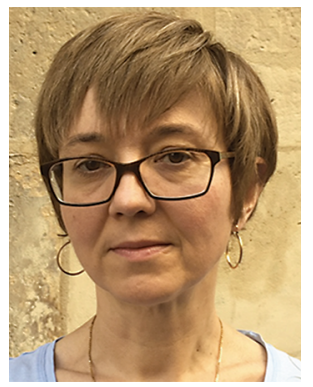

Liliana Borcea

\section{Credits}

Figures 1-2, 4, and 6 are courtesy of Jörn Zimmerling.

Figures 3 and 5 are reprinted with permission of IOP Publishing in the format Journal/magazine via Copyright Clearance Center from: L. Borcea, G. C. Papanicolau, and C. Tsogka. Adaptive interferometric imaging in clutter and optimal illumination. Inverse Problems, 22(4):1405, 2006, doi:10.1088/0266-5611/22/4/016

Photo of author is courtesy of Lucian Borcea.

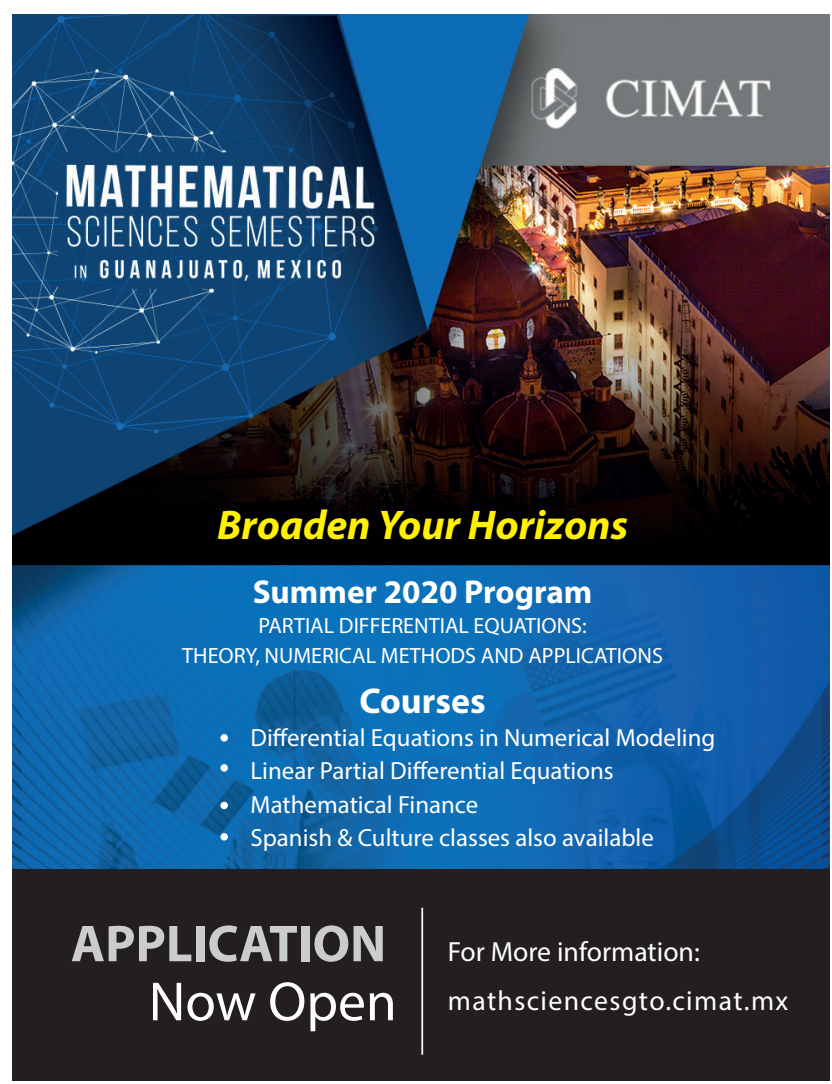

